\title{
Morphological classification and clinical significance of medial malleolus based on computed tomography three-dimensional reconstruction
}

\author{
L. Xin ${ }^{1}$, K. $\operatorname{Liu}^{2}$ *, B. He *, M. Chen ${ }^{*}$, B. Tang ${ }^{1}$, C. Tang ${ }^{1}$, L. Zhang 4, 5, 6, 7 * \\ ${ }^{1}$ Department of Orthopaedics, Jiang'an Hospital of Traditional Chinese Medicine, Yibin Sichuan, China \\ 2Department of Radiology, Yibin Third People's Hospital, Yibin Sichuan, China \\ ${ }^{3}$ Department of Internal Medicine, Jiang'an Hospital of Traditional Chinese Medicine, Yibin Sichuan, China \\ ${ }^{4}$ Department of Orthopaedics, Affiliated Traditional Chinese Medicine Hospital of Southwest Medical University, \\ Luzhou Sichuan, China \\ ${ }^{5}$ Centre for Orthopaedic Diseases Research, Affiliated Traditional Chinese Medicine Hospital of Southwest \\ Medical University, Luzhou Sichuan, China \\ ${ }^{6}$ Expert Workstation in Luzhou, Luzhou Sichuan, China \\ ${ }^{7}$ Clinical Base of Affiliated Traditional Chinese Medicine Hospital of Southwest Medical University, Guangdong Province \\ Medical 3D Printing Application Transformation Engineering Technology Research Centre, Luzhou Sichuan, China
}

[Received: 5 September 2021; Accepted: 10 December 2021; Early publication date: 16 December 2021]

Background: Internal malleolus fractures and postoperative functional limitations are serious complications of deltoid ligament repair, reconstruction, while studies on conducting beak. Anatomical structure classification of medial malleolus at home and abroad is reported rarely. Hence, this morphological study is mainly designed to investigate the anatomical morphological classification and clinical significance of medial malleolus based on computed tomography (CT) three-dimensional reconstruction.

Materials and methods: From October 2018 to January 2021, 373 patients who underwent CT examination of malleolus medialis joint in the Jiang'an Hospital of Traditional Chinese Medicine were observed. The medial malleolus was observed and classified; then, geometric parameters were measured according to different medial malleolus types.

Results: According to the results of 373 cases, medial malleolus can be divided into four types: omega type (66\%), radical sign type (16\%), inverted triangle type (14\%), and wave type (4\%).

Conclusions: There are four main shapes: omega, inverted triangle, radical sign, and wave in the medial malleolus of all normal ankles. The measurement of medial malleolus parameters according to medial malleolus in different shapes was of importance to guide smooth operation of medial malleolus fixation and deltoid ligament reconstruction and epidemiological. (Folia Morphol 2023; 82, 1: 176-182)

Key words: medial malleolus, deltoid ligament, anatomical morphology, computed tomography three-dimensional reconstruction

Address for correspondence: Dr. L. Zhang, Department of Orthopaedics, Affiliated Traditional Chinese Medicine Hospital of Southwest Medical University, Luzhou, China, e-mail: zhanglei870722@126.com; Dr. L. Xin, Department of Orthopaedics, Jiang'an Hospital of Traditional Chinese Medicine, Yibin Sichuan, China, e-mail: 1258356206@qq.com

*These authors contributed equally to this work.

This article is available in open access under Creative Common Attribution-Non-Commercial-No Derivatives 4.0 International (CC BY-NC-ND 4.0) license, allowing to download articles and share them with others as long as they credit the authors and the publisher, but without permission to change them in any way or use them commercially. 


\section{INTRODUCTION}

The deltoid ligament, originates from the lower margin of the medial malleolus, splits and terminates at the scaphoid, talus and anteromedial calcaneus. It is divided into deep and superficial layers $[5,25]$. The superficial ligament consists of 4 bundles that are tibial navicular ligament, tibial spring ligament, tibial calcaneal ligament and tibial talus ligament in sequence. The deep layer consists of the anterior and posterior tibial talus ligaments $[8,15]$, and the construction is to keep the joint stable by limiting hyperpronation of the talus. The deltoid ligament is the main medial stabilizer of the ankle joint, preventing valgus and anterior talus displacement, and it is also the most important structure to prevent lateral displacement $[2,19,23]$. Overall, the deltoid ligament provides stability for the tibial talo-calcaneal complex. After injuries of deltoid ligament, the stability is weakened, as time goes by, gradually leading to a decrease in contact with the articular surface of the talus to the contralateral, also leading to an increase in contralateral stress on the articular surface $[12$, $13,17]$ and an increase in joint wear. Studies have shown that the displacement of $1 \mathrm{~mm}$ will lose $42 \%$ of the relative contact area and increase $30 \%$ of the stress $[2,7,16,22]$. Therefore, the triangular ligament should be repaired according to the anatomical and biomechanical characteristics in order to maximise the recovery of its biomechanical structure and tensile strength, and consequently the stability of the ankle, and to improve patient satisfaction after surgery.

The incidence of simple medial malleolus fracture accounts for $7 \%$ of all ankle fractures, about 3 times that of the lateral malleolus fractures $[1,14]$, while the deltoid ligament injury accounts for $6.6-15 \%$ of ankle injuries [9]. In clinical practice, fracture reduction and internal fixation is one of the most frequently surgery methods used for medial malleolus fracture at present. In the case of deltoid ligament injury, deltoid ligament reconstruction is the most common choice in foreign countries $[10,21]$, and repair of the triangular ligament is the most common choice in China $[3,11]$. In recent years, reconstruction of the triangular ligament has been used in the treatment of flat foot [20] and has received good clinical results. During the fracture reduction and internal fixation reconstruction of the deltoid ligament, it is necessary to recovery the medial malleolus, and the selection of the location, size and internal thickness of the tunnel determines the success of the operation [18]. Superficial ligament is visible intraoperatively, and the hole location is easy to be determined, which has been studied at home and abroad; because of the smaller malleolus medialis and complex surrounding anatomical structures, the deep ligaments itself is difficult to be positioned accurately [24], especially in the case of minimally invasive surgery in which the vision is limited. When drilling easy position offset internal malleolus fracture and postoperative functional limitations has become serious complications of deltoid ligament repair and reconstruction, and literatures conducting on beak. Anatomical structure classification of medial malleolus at home and abroad is reported rarely.

Therefore, the purpose of this study is to observe the anatomy of malleolus medialis, and to discuss the indications and contraindications of deltoid ligament reconstruction based on computed tomography (CT) three-dimensional (3D) reconstruction. It can also provide the effective anatomy basis for the deltoid ligament reconstruction, in order to reduce the risk of operation and to lower the incidence of postoperative complications.

\section{MATERIALS AND METHODS}

\section{Materials}

All patients undergoing 3D CT scan were screened from October 2018 to January 2021. A total of 373 subjects aged between 19 and 66 years were included in this study. There were 198 males and 175 females. There were 177 cases on the left side and 196 cases on the right side, with a mean age of $46.04 \pm 10.35$ years.

Inclusion criteria: (1) The development of tibia, medial malleolus and deltoid ligament was normal; (2) There was no history of medial malleolus or tibia fracture, and no history of deltoid ligament injury.

Exclusion criteria: (1) malformation of medial malleolus, talus or deltoid ligament; (2) A history of ankle surgery.

\section{Methods}

Scans of mid-lower tibia to calcaneus were obtained using a 16-row 128-slice spiral Siemens CT scanner, with patients lying in supine position. The scanned images were transferred to simulation software for 3D reconstruction and $1 \mathrm{~mm}$ thin sections.

Patients because of own or external damage, such as car accident sprain, no obvious cause of pain and other reasons below the knee and leg pain related symptoms need the $\mathrm{CT}$ scans. Then preliminary screening of the medial malleolus of all patients 

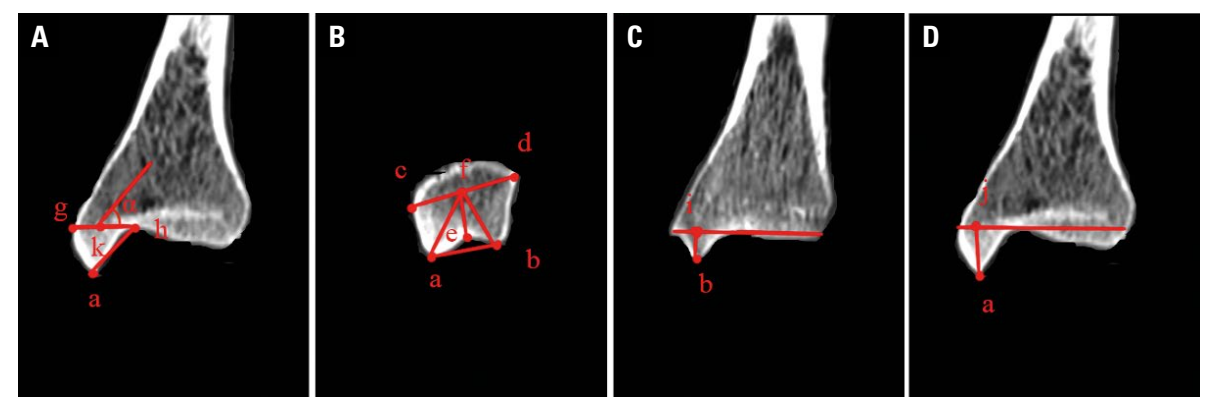

Figure 1. A-D. Morphological measurements were described as follows; ab — sagittal view, medial malleolus, distance between anterior and posterior colliculus (point $a$ is the medial malleolus anterior colliculus, point $b$ is the medial malleolus posterior colliculus); $c d$ - medial malleolus anteroposterior to lips farthest distance (point $\mathrm{c}$ is the anterior labial protrusion of the medial malleolus; point $\mathrm{d}$ is the most protruding point of the posterior lip of the medial malleolus); ef — the distance from the anteriorly hump point of the medial malleolus to the midpoint of the anteriorly lip of the medial malleolus (point $\mathrm{e}$ is the anterior and posterior dune depression of the medial malleolus, point $\mathrm{f}$ is the midpoint of the distance between the front and back lips); bf — distance from posterior colliculus of medial malleolus to centre of anterior and posterior labial margin of medial malleolus; af — distance between medial malleolus anterior colliculus and medial malleolus anterior labial margin centre; bi — on the sagittal plane, the distance from posterior colliculus to tibial (point $i$ is the point from point $B$ where it intersects the vertical line of the articular surface of the tibial talus); $\angle \alpha$ - the angle between the midline and the horizontal line of the internal ankle joint; aj - distance from anterior colliculus to tibial joint surface (point $j$ is the point from point a where it intersects the vertical line of the articular surface of the tibial talus).
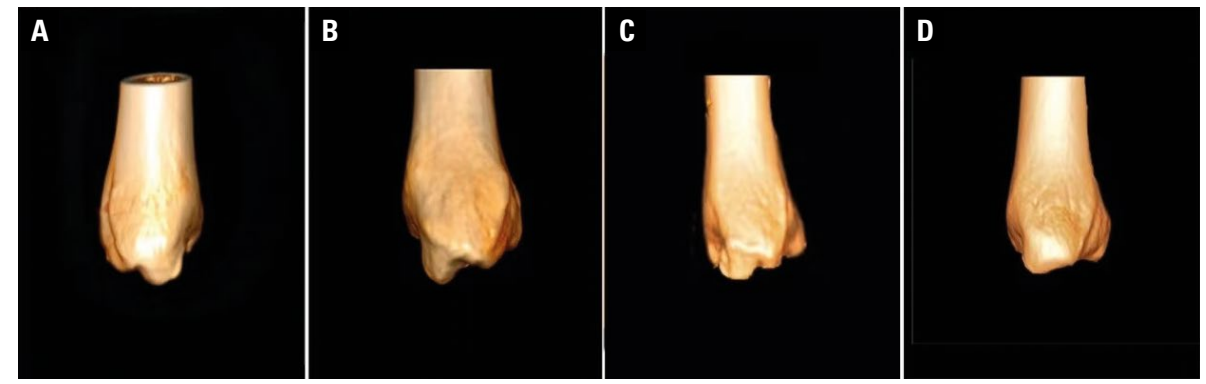

Figure 2. A-D. The types of the medial malleolus.

undergoing 3D reconstruction was performed by two radiologists. Patients with fractures, malformations, surgical history, or whose age was not in the range of 19-66 years were excluded from the analysis. The medial malleolus was then carefully observed for any other inconspicuous symptoms. Any doubts regarding the classification were discussed and a joint decision was made. Then, geometric parameters were measured, taking the average of the final results. Otherwise, doubts were resolved by the director of radiology department. All the observers have worked in professional imaging for more than 10 years.

The shape of the medial malleolus was first observed and classified on the CT 3D reconstruction. Second, the clearest and most complete medial malleolus layer in the coronal and sagittal planes was selected by means of $\mathrm{CT}$ to measure geometric parameters and their adjacent structures, as it was shown in Figure 1.

\section{Statistical analyses}

Statistical analyses were performed using SPSS (version 20.0; IBM Corp.). All the measured data were showed by mean \pm standard deviation. The measured geometric parameters corresponding to the type of medial malleolus were analysed by one-way analysis of variance (LSD-t). $P<0.05$ was considered to indicate a statistically significant difference.

\section{RESULTS}

According to the shape of the medial malleolus, it can be divided into omega type, inverted triangle type, wave type and radical sign type, as shown in Figure 2.

In the 3D reconstruction of adult medial malleolus, $248(66 \%)$ cases were omega type, $52(14 \%)$ cases were inverted triangle type, $12(4 \%)$ cases were wave type and $61(16 \%)$ cases were radical sign type. The measured geometric parameters corresponding to each type of medial malleolus were as shown in Table 1. There were significant differences in the af $(p=0.000)$ 
Table 1. Measured values of the medial malleolus based on sides of the body and sexes

\begin{tabular}{lcccc}
\hline Distribution & Male & Female & Left & Right \\
\hline Numbers & $198(53.08 \%)$ & $175(46.92 \%)$ & $183(49.06 \%)$ & $190(50.94 \%)$ \\
ab $[\mathrm{cm}]$ & $14.73 \pm 2.90$ & $15.04 \pm 3.15$ & $14.97 \pm 3.06$ & $14.78 \pm 2.98$ \\
cd $[\mathrm{cm}]$ & $27.11 \pm 3.87$ & $27.60 \pm 3.84$ & $27.53 \pm 3.87$ & $27.16 \pm 3.85$ \\
ef $[\mathrm{cm}]$ & $11.04 \pm 3.44$ & $11.51 \pm 3.21$ & $11.06 \pm 3.32$ & $11.45 \pm 3.36$ \\
af $[\mathrm{cm}]$ & $16.45 \pm 3.62$ & $16.67 \pm 3.45$ & $16.36 \pm 3.52$ & $16.74 \pm 3.58$ \\
bf $[\mathrm{cm}]$ & $14.79 \pm 3.51$ & $14.66 \pm 3.14$ & $14.92 \pm 2.34$ & $14.73 \pm 3.26$ \\
aj $[\mathrm{cm}]$ & $12.71 \pm 1.15$ & $11.97 \pm 1.61$ & $12.63 \pm 1.45$ & $12.11 \pm 1.86$ \\
bi $[\mathrm{cm}]$ & $5.97 \pm 2.05$ & $5.79 \pm 1.93$ & $5.75 \pm 1.95$ & $6.03 \pm 2.04$ \\
$\left.\angle \alpha{ }^{\circ}\right]$ & $45.04 \pm 7.14$ & $45.59 \pm 7.21$ & $45.12 \pm 7.13$ & $45.47 \pm 7.23$ \\
\hline
\end{tabular}

Table 2. Morphological measurements in four types

\begin{tabular}{lcccc}
\hline & Inverted triangle type & Omega type & Radical sign type & Wave type \\
\hline Numbers & $52(14 \%)$ & $248(66 \%)$ & $61(16 \%)$ & $12(4 \%)$ \\
ab $[\mathrm{cm}]$ & $15.23 \pm 3.87^{\mathrm{bc}}$ & $14.73 \pm 2.89^{\mathrm{c}}$ & $16.50 \pm 2.35^{\mathrm{c}}$ & $11.42 \pm 0.61$ \\
$\mathrm{~cd}[\mathrm{~cm}]$ & $27.45 \pm 3.86^{\mathrm{bc}}$ & $25.82 \pm 3.98^{\mathrm{b}}$ & $29.44 \pm 2.22$ & $27.59 \pm 3.64$ \\
ef $[\mathrm{cm}]$ & $11.19 \pm 3.73^{\mathrm{bc}}$ & $11.26 \pm 3.36^{\mathrm{bc}}$ & $12.51 \pm 2.62^{\mathrm{c}}$ & $8.31 \pm 3.14$ \\
af $[\mathrm{cm}]$ & $18.31 \pm 2.54^{\mathrm{ac}}$ & $16.39 \pm 3.63^{\mathrm{b}}$ & $17.94 \pm 2.47^{\mathrm{c}}$ & $14.44 \pm 4.51$ \\
bf $[\mathrm{cm}]$ & $14.73 \pm 3.33^{\mathrm{c}}$ & $14.99 \pm 3.45^{\mathrm{bc}}$ & $13.59 \pm 2.34^{\mathrm{c}}$ & $11.35 \pm 4.35$ \\
aj $[\mathrm{cm}]$ & $12.39 \pm 1.85^{\mathrm{c}}$ & $12.61 \pm 1.78$ & $12.25 \pm 1.63^{\mathrm{c}}$ & $8.15 \pm 2.12$ \\
bi $[\mathrm{cm}]$ & $5.21 \pm 2.26^{\mathrm{ac}}$ & $6.06 \pm 1.91$ & $5.57 \pm 2.06^{\mathrm{c}}$ & $6.98 \pm 1.14$ \\
$\angle \alpha\left[{ }^{\circ}\right]$ & $45.36 \pm 8.09^{\mathrm{c}}$ & $45.93 \pm 6.43^{\mathrm{bc}}$ & $43.43 \pm 8.30^{\mathrm{c}}$ & $38.07 \pm 5.55$ \\
\hline
\end{tabular}

${ }^{a} p<0.05$ vs. omega type, ${ }^{b} p<0.05$ vs. radical sign type, ${ }^{c} p<0.05$ vs. wave type

and bi ( $p=0.005$ ) between omega type and inverted triangle type. There were statistical differences in the $a b(p=0.022), c d(p=0.005)$, and ef ( $p=0.035$ ) between inverted triangle type and radical sign type $(p<0.05)$. There were statistical differences in the $a b(p=0.000)$, ef $(p=0.007)$, af $(p=0.000)$, bf $(p=0.002)$, aj $(p=0.000)$, bi $(p=0.005)$, and $\angle \alpha(p=0.001)$ between inverted triangle type and wave type. There were statistical differences in the cd $(p=0.000)$, ef $(p=0.008)$, af $(p=0.001)$, bf $(p=0.003)$, and $\angle \alpha(p=0.013)$ between omega type and radical sign type $(p<0.05)$. There were statistical differences in the $a b(p=0.000)$, ef $(p=0.003)$, bf $(p=0.000)$, and $\angle \alpha(p=0.000)$ between omega type and wave type. There were statistical differences in the $c d(p=0.000)$, ef $(p=0.008)$, af $(p=0.001)$, bf ( $p=0.003)$, and $\angle \alpha(p=0.013)$ between omega type and radical sign type $(p<0.05)$. There were statistical differences in the $a b(p=0.000)$, ef $(p=0.000)$, af $(p=0.001)$, bf $(p=0.032)$, aj $(p=0.000)$, bi $(p=0.024)$, and $\angle \alpha(p=0.016)$ between wave type and radical sign type. The measured geometric parameters corresponding to each type of medial malleolus were not significantly different between left and right, as shown in Table 2.

\section{DISCUSSION}

Ankle is the weight-bearing joint closest to the ground and the most important weight-bearing joint in the body [4]. The stability of the ankle joint plays a very important role in daily exercise and life. With the development of society and the improvement of people's living standard, ankle joint injury mostly occurs in people with strong athletic ability. Eighty five per cent of patients suffer from ankle sprain, which is one of the most common sports injuries; $40 \%$ of patients may have residual chronic symptoms, and approximately $5 \%$ of ankle injuries are associated with deltoid ligament injuries. However, recent studies have found that $40 \%$ to $73 \%$ of patients with chronic lateral ankle instability may suffer from deltoid ligament injuries [6]. The deltoid ligament is the strongest ligament in the ankle joint, and its main function is to prevent the ankle from valgus. It starts from the 
medial malleolus, fanning down into a bundle and terminates in the scaphoid, talus, and calcaneus. For patients with ligament injury, ligament reconstruction can restore the stability of the ankle joint $[10,21]$.

Therefore, the treatment of deltoid ligament injury is the key to restore the function of the ankle joint. At present, the treatment of deltoid ligament injury in China mainly uses the suture anchor to repair the ligament $[3,11]$. In foreign countries, ligament reconstruction is the main therapeutic method $[10,21]$. Whether it is transosseous suture or wire repair, suture with suture anchors or newly developed ligament reconstruction, a borehole is required to establish the hole. The key to reconstruction lies in the diameter and location of the bone tunnel [18]. Improper pore size, position or drilling location can easily lead to unbefitting fixation of postoperative implants, resulting in surgical failure, redislocation, clavicle and coracoid process clefts, and bone fractures. At present, there are few clinical studies on indications and contraindications of the deltoid ligament repairing or reconstruction under different coracoid process shapes, which is also one of the reasons for the high risk of surgical failure and multiple postoperative complications. Therefore, the anatomical morphology of medial malleolus and the anatomical parameters related to the repair or reconstruction of the deltoid ligament need to be studied urgently.

The purpose of this study was to investigate the morphology of the medial malleolus and provide anatomic basis and predictive value for the treatment and surgical guidance of ankle joint injuries. In this study, the medial malleolus could be divided into four different types, among which the omega type accounted for the highest proportion. Most of the geometrical parameters and the anatomical parameters of their attachment points are different under diverse medial malleolus. Therefore, for the ankle injury patients with different medial malleolus shapes, individualised treatment should be paid attention to, so as to improve the success rate of internal fixation of medial malleolus fracture reduction, deltoid ligament repair or reconstruction, and reduce postoperative complications, especially the incidence of medial malleolus fracture. In addition, the attachment points of the deltoid ligament were studied to guide clinicians in selecting the location of bone tunnel drilling.

Among the four shapes of medial malleolus, af was the longest and aj was longer while bi was shorter corresponding to type $\mathrm{v}$. It showed that the anterior and posterior colliculus were diagonal, so the contact area between the V-shaped deltoid ligament and the medial malleolus was minor or even narrower, preventing excessive movement and other injuries of deltoid ligament caused by unbalanced factors or medial malleolus fractures. The anterior colliculus of the inverted-triangle medial malleolus is quite narrow, and theoretically the drilling location should be far away from the anterior colliculus, but the bi of the four types of medial malleolus is also shorter. Failure to drill in front of the medial malleolus when the bone tunnel is drilled may result in changes in biomechanics after deltoid ligament repair. Therefore, the inverted-triangle medial malleolus should not be fixed with the deltoid ligament of the tunnel, and the medial malleolus upward wire anchor should be considered. The corresponding ab, $c d$ and ef of the radical sign type are the longest, while the aj, bi and $\angle \alpha$ are minor. The radical sign type is similar to the inverted triangle type, and the two joints are oblique and the distance is large. The corresponding population should take protective measures to avoid deltoid ligament injury and medial malleolus fracture caused by imbalanced factors or strenuous exercise. Therefore, when the deltoid ligament corresponding to the radical sign type is broken or the medial malleolus fractures, and the bone volume of medial malleolus of the radical sign type is small, the probability of medial malleolus fractures increases during drilling. In addition, the anterior colliculus is short and the annular structure is prone to slippage. Therefore, when the deltoid ligament corresponding to the medial malleolus of radical sign type is broken or the medial malleolus fractures, it is not appropriate to use deltoid ligament reconstruction, but suture anchor fixation can be adopted. Except the cd and $\mathrm{bi}$, medial malleolus of the wave type are the longest, while the ab, ef, af, bf, aj and $\angle \alpha$ are the smallest. The distance between the two colliculi is short, showing a relatively horizontal line. The distance between the two mounds, the upper and lower lips of the medial malleolus, is the smallest, and the overall height between the two lips, the upper and lower mounds, is the smallest. Theoretically, the contact area between the medial malleolus and the deltoid ligament of the wave type is relatively wide, and there is more bone in the medial malleolus hilum, and the amount of bone in the hilum of the medial malleolus is more, and the anatomical structure is appropriate. Therefore, deltoid ligament reconstruction can be recommend- 
ed for the treatment of deltoid ligament injury. Of the omega type, aj and bi were the largest, but the distance was small, cd was the smallest, ab and ef were smaller. Theoretically, the distance between the two colliculi and grooves to the two lips is small, and the volume of the medial malleolus bone is larger, so the anatomical structure is appropriate. Therefore, it is recommended to carry out deltoid ligament reconstruction to treat the deltoid ligament injury. The ef of inverted triangle type was $11.19 \pm 3.73 \mathrm{~cm}$, and that of the omega type was $11.26 \pm 3.36 \mathrm{~cm}$. For deltoid ligament reconstruction or internal fixation, the locations of inverted triangle type and omega type tunnels were approximately the same. The measure of $\angle \alpha$ of wave type was $38.07 \pm 5.55^{\circ}$, omega type was $45.93 \pm 6.43^{\circ}$, inverted triangle type was $45.36 \pm 8.09^{\circ}$, radical sign type was $43.43 \pm 8.30^{\circ}$. The measures of $\angle \alpha$ of wave type and radical sign type were smaller. The medial malleolus was slightly horizontal, and the $\angle \alpha$ of omega type and inverted triangle type were larger, which were more vulnerable to external force injury and fracture. Wear high heels less in daily life, because the external force collision can affect the selection of fixation angle and direction of clinical internal fixators. Above all, different treatment plans and materials should be used to repair medial malleolus fractures and deltoid ligaments of different shapes.

\section{Limitations of the study}

There are some limitations in this study. Only patients from southwest China were collected, mostly from rural areas, and the sample size was small. In this study, further biomechanical studies could be performed to investigate whether the geometric parameters corresponding to the w-shaped and $\mathrm{v}$-shaped deltoid ligament are shorter, thinner or easier to break. In this study, only the relevant classification and anatomical data of the medial malleolus and the possible mechanism of related diseases were discussed, and further data tracking and improvement are needed.

\section{CONCLUSIONS}

The medial malleolus of all normal ankles has four main shapes: omega, inverted triangle, radical sign, and wave. Medial malleolus of omega type and wave type are more suitable for internal fixation, ligament repair and reconstruction, while inverted triangle type and radical sign type have the narrowest colliculi. Therefore, deltoid ligament reconstruction is generally not appropriate, and internal fixation with thread anchors is preferred. Wave-shaped and radical-sign-shaped medial malleolus have a smaller angle, and the medial malleolus is slightly inclined and horizontal. Omega and inverted triangle have a larger medial malleolus angle, which is more vulnerable to external force and violence injury resulting in fracture. Determining the corresponding measurement parameters according to medial malleolus in different shapes is of significance to guide smooth operation of medial malleolus fixation and deltoid ligament reconstruction surgery.

\section{Acknowledgements}

The authors wanted to show their gratitude to the imaging department in Jiang'an Hospital of Traditional Chinese Medicine that provided the samples.

\section{Funding}

The study received grants from the National Natural Science Foundation of China (No. 82004458); Traditional Chinese Medicine Research Project of Sichuan Traditional Chinese Medicine Administration (No. 2020JC0138); Health Commission of Sichuan Province Science and Research Project (No. 20PJ143); Luzhou People's Government-Southwest Medical University Shi-zhen Zhong Academician Talent Team Sub-project (2018zszysrctdxm), and Southwest Medical University Research Project (2020ZRQNA045).

\section{Conflict of interest: None declared}

\section{REFERENCES}

1. Aitken SA, Johnston I, Jennings AC, et al. An evaluation of the Herscovici classification for fractures of the medial malleolus. Foot Ankle Surg. 2017; 23(4): 317-320, doi: 10.1016/j.fas.2016.10.003, indexed in Pubmed: 29202995.

2. Alshalawi S, Galhoum AE, Alrashidi Y, et al. Medial ankle instability: the deltoid dilemma. Foot Ankle Clin. 2018; 23(4): 639-657, doi: 10.1016/j.fcl.2018.07.008, indexed in Pubmed: 30414658.

3. Bastias GF, Filippi J. Acute deltoid ligament repair in ankle fractures. Foot Ankle Clin. 2020; 25(4): 597-612, doi: 10.1016/j.fcl.2020.08.009, indexed in Pubmed: 33543718.

4. Bruno F, Arrigoni F, Palumbo P, et al. Weight-bearing MR imaging of knee, ankle and foot. Semin Musculoskelet Radiol. 2019; 23(6): 594-602, doi: 10.1055/s-0039-1697940, indexed in Pubmed: 31745949.

5. Campbell KJ, Michalski MP, Wilson KJ, et al. The ligament anatomy of the deltoid complex of the ankle: a qualitative and quantitative anatomical study. J Bone Joint Surg Am. 
2014; 96(8): e62, doi: 10.2106/JBJS.M.00870, indexed in Pubmed: 24740670.

6. Cao $Y$, Hong $Y, X u Y$, et al. Surgical management of chronic lateral ankle instability: a meta-analysis. J Orthop Surg Res. 2018; 13(1): 159, doi: 10.1186/s13018-018-0870-6, indexed in Pubmed: 29940985.

7. Clanton TO, Williams BT, Backus JD, et al. Biomechanical analysis of the individual ligament contributions to syndesmotic stability. Foot Ankle Int. 2017; 38(1): 66-75, doi: 10.1177/1071100716666277, indexed in Pubmed: 27681857.

8. Crim J. Medial-sided ankle pain: deltoid ligament and beyond. Magn Reson Imaging Clin N Am. 2017; 25(1): 63-77, doi: 10.1016/j.mric.2016.08.003, indexed in Pubmed: 27888852.

9. Debieux P, Wajnsztejn A, Mansur N. Epidemiology of injuries due to ankle sprain diagnosed in an orthopedic emergency room. Einstein (São Paulo). 2019; 18, doi: 10.31744/einstein_journal/2020ao4739.

10. Hajewski CJ, Duchman K, Goetz J, et al. Anatomic syndesmotic and deltoid ligament reconstruction with flexible implants: a technique description. lowa Orthop J. 2019; 39(1): 21-27, indexed in Pubmed: 31413670.

11. Hardy MA, Connors JC, Zulauf EE, et al. Acute deltoid ligament repair in ankle fractures: five-year follow-up. Clin Podiatr Med Surg. 2020; 37(2): 295-304, doi: 10.1016/j. cpm.2019.12.004, indexed in Pubmed: 32146985.

12. Hartford JM, Gorczyca JT, McNamara JL, et al. Tibiotalar contact area. Contribution of posterior malleolus and deltoid ligament. Clin Orthop Relat Res. 1995(320): 182-187, indexed in Pubmed: 7586825.

13. Kobayashi T, Suzuki E, Yamazaki N, et al. In vivo talocrural joint contact mechanics with functional ankle instability. Foot Ankle Spec. 2015; 8(6): 445-453, doi: 10.1177/1938640015585967, indexed in Pubmed: 25956876.

14. Kusnezov NA, Eisenstein ED, Diab N, et al. Medial malleolar fractures and associated deltoid ligament disruptions: current management controversies. Orthopedics. 2017; 40(2): e216-e222, doi: 10.3928/01477447-20161213-02, indexed in Pubmed: 27992638.

15. Lee S, Lin J, Hamid KS, et al. Deltoid Ligament Rupture in Ankle Fracture: Diagnosis and Management. J Am Acad Orthop Surg. 2019; 27(14): e648-e658, doi: 10.5435/ JAAOS-D-18-00198, indexed in Pubmed: 30475279.

16. Liu C, Wang Y, Li Z, et al. Tibiofemoral joint contact area and stress after single-bundle anterior cruciate ligament reconstruction with transtibial versus anteromedial portal drilling techniques. J Orthop Surg Res. 2018; 13(1): 247-255, doi: 10.1186/s13018-018-0956-1, indexed in Pubmed: 30286774.

17. Macko VW, Matthews LS, Zwirkoski P, et al. The joint-contact area of the ankle. The contribution of the posterior malleolus. J Bone Joint Surg Am. 1991; 73(3): 347-351, indexed in Pubmed: 2002072.

18. Michels F, Guillo S, Vanrietvelde F, et al. How to drill the talar tunnel in ATFL reconstruction? Knee Surg Sports Traumatol Arthrosc. 2016; 24(4): 991-997, doi: 10.1007/ s00167-016-4018-0, indexed in Pubmed: 26856316.

19. Milner CE, Soames RW. The medial collateral ligaments of the human ankle joint: anatomical variations. Foot Ankle Int. 1998; 19(5): 289-292, doi: 10.1177/107110079801900504, indexed in Pubmed: 9622418.

20. Nery C, Lemos AV, Raduan F, et al. Combined spring and deltoid ligament repair in adult-acquired flatfoot. Foot Ankle Int. 2018; 39(8): 903-907, doi: 10.1177/1071100718770132, indexed in Pubmed: 29658814

21. Pisanu F, Ortu S, Corda M, et al. Deltoid ligament reconstruction with autologous gracilis tendon in chronic medial ankle instability after ankle fracture surgery: A case report. Foot (Edinb). 2021; 49: 101714, doi: 10.1016/j. foot.2020.101714, indexed in Pubmed: 33036835.

22. Ramsey PL, Hamilton W. Changes in tibiotalar area of contact caused by lateral talar shift. J Bone Joint Surg Am. 1976; 58(3): 356-357, indexed in Pubmed: 1262367.

23. Salameh M, Alhammoud A, Alkhatib N, et al. Outcome of primary deltoid ligament repair in acute ankle fractures: a meta-analysis of comparative studies. Int Orthop. 2020; 44(2): 341-347, doi: 10.1007/s00264-019-04416-9, indexed in Pubmed: 31776609.

24. Savage-Elliott I, Murawski CD, Smyth NA, et al. The deltoid ligament: an in-depth review of anatomy, function, and treatment strategies. Knee Surg Sports Traumatol Arthrosc. 2013; 21(6): 1316-1327, doi: 10.1007/s00167012-2159-3, indexed in Pubmed: 22878436.

25. Stufkens SAS, van den Bekerom MPJ, Knupp M, et al. The diagnosis and treatment of deltoid ligament lesions in supination-external rotation ankle fractures: a review. Strategies Trauma Limb Reconstr. 2012; 7(2): 73-85, doi: 10.1007/ s11751-012-0140-9, indexed in Pubmed: 22767333. 